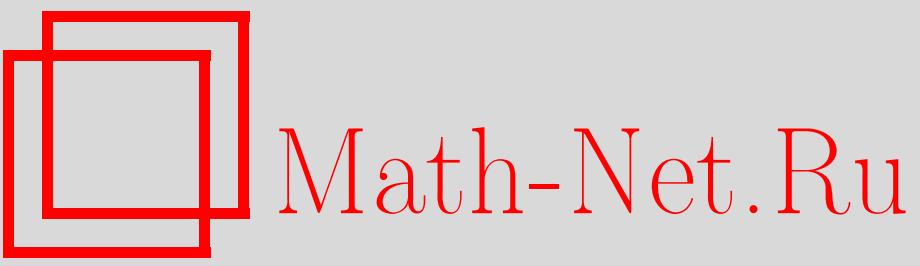

У. А. Розиков, О счетно-периодических гиббсовских мерах модели Изинга на дереве Кэли, ТМФ, 2002, том 130, номер 1, 109-118

DOI: https://doi.org/10.4213/tmf293

Использование Общероссийского математического портала Math-Net.Ru подразумевает, что вы прочитали и согласны с пользовательским соглашением

http://www.mathnet.ru/rus/agreement

Параметры загрузки:

IP: 3.80 .181 .102

26 апреля 2023 г., 13:41:17 
ТЕОРЕТИЧЕСКАЯ

И МАТЕМАТИЧЕСКАЯ

ФИЗИКА

Том 130, № 1

январь, 2002

(C) 2002 г.

У.А. Розиков*

\section{О СЧЕТНО-ПЕРИОДИЧЕСКИХ ГИББСОВСКИХ МЕРАХ МОДЕЛИ ИЗИНГА НА ДЕРЕВЕ КЭЛИ}

Описан достаточно широкий класс нормальных делителей бесконечного индекса группового представления дерева Кэли, изучена структура разбиений дерева Кэли относительно любого нормального делителя бесконечного индекса. Для конкретного нормального делителя бесконечного индекса доказано, что существуют три периодические и несчетное число непериодических гиббсовских мер для неоднородной модели Изинга.

\section{1. ВВЕДЕНИЕ}

В основе теории гиббсовских мер лежит формальное выражение, называемое гамильтонианом. Одна из основных проблем заключается в описании предельных гиббсовских мер, отвечающих данному гамильтониану.

В обшем случае сушествование предельных гиббсовских мер для достаточно широкого класса гамильтонианов доказано Добрушиным (см., например, [1]). Но задача описания множества всех предельных гиббсовских мер для конкретного гамильтониана весьма трудная, и окончательные результаты получаются лишь при малых $\beta=1 / T$ ( $T$ > 0 - температура) (см. [1]-[3]). Трудность задачи также зависит от множества $\Phi$ значений спина и от структуры графа, на котором рассматривается гамильтониан.

Для широкого класса счетных множеств $S$ задача сушествования фазовых переходов, т.е. неединственность предельных гиббсовских мер, изучена [2] и найдены необходимые и достаточные условия на языке корреляционных функций.

Много работ посвяшено описанию множества предельных гиббсовских мер на $Z^{d}$, $d \in N=\{1,2,3, \ldots\}$, где $Z^{d}$ - целочисленная решетка (см., например, [1]-[4]), и на дереве Кэли $\Gamma^{k}, k \geqslant 1$, где $\Gamma^{k}$ есть бесконечное дерево, т.е. граф без циклов, из каждой вершины которого выходят ровно $k+1$ ребер (см., например, [5]-[12]), в случае, когда радиус взаимодействия конечный. В этих работах в основном описаны множества трансляционно-инвариантных и периодических гиббсовских мер относительно подгруппы $Z_{1}^{d} \subset Z^{d}$ конечного индекса (соответственно относительно подгруппы конечного индекса группового представления дерева Кэли).

Задача описания периодических гиббсовских мер относительно подгрупп бесконечного индекса ранее не рассматривалась, так как в этом случае возникает система счет-

${ }^{*}$ Институт математики им. В.И. Романовского АН РУз, Ташкент, Республика Узбекистан. E-mail: root@im.tashkent.su 
ного числа уравнений (относительно корреляционных функций и некоторых функций, связанных с ними) с бесконечным числом неизвестных.

Известно, что дерево Кэли $\Gamma^{k}, k \geqslant 1$, представляется как группа $G_{k}$, являющаяся свободным произведением $k+1$ циклических групп второго порядка с образуюшими $a_{1}, a_{2}, \ldots, a_{k+1}[11]$.

Заметим, что группа $G_{k}, k \geqslant 1$, некоммутативная, а $Z^{d}$ коммутативная. Для построения периодических гиббсовских мер на дереве Кэли необходимо описать нормальные делители группы $G_{k}$. Достаточно широкий класс нормальных делителей конечного индекса построен в работе [5]. Структура разбиений дерева Кэли относительно нормальных делителей конечного индекса изучена в работе [6].

В настояшей работе рассматривается неоднородная модель Изинга на дереве $\Gamma^{2}$. Основная цель работы - описать для этой модели периодические меры Гиббса (отличные от периодических мер Гиббса, соответствующих подгруппам конечного индекса). В разделе 2 описывается класс некоторых нормальных делителей бесконечного индекса и изучается структура разбиений дерева Кэли относительно любого нормального делителя бесконечного индекса. Это позволило нам рассмотреть задачу описания периодических гиббсовских мер. Эти меры, называемые счетно-периодическими, строятся в разделе 3 . В разделе 4 с помощью мер, построенных в разделе 3 , описывается новый класс предельных гиббсовских мер, которые отличаются от ранее известных (см. [5]-[8]).

\section{2. НОРМАЛЬНЫЕ ДЕЛИТЕЛИ БЕСКОНЕЧНОГО ИНДЕКСА ГРУППЫ $G_{k}$}

Пусть $G_{k}$ - свободное произведение $k+1$ циклических групп второго порядка с образуюшими $a_{1}, a_{2}, \ldots, a_{k+1}$.

ПРЕДЛОЖЕНИЕ 1 [11]. Существует взаимно однозначное соответствие между множеством вершин $V$ дерева Кәли $\Gamma^{k}$ и әруппой $G_{k}$.

Для описания нормальных делителей этой групшы нам потребуется следуюшее

ПРЕДЛОЖЕНИЕ 2 [13]. Пусть $G u G^{*}-$ произвольные группы и $\varphi$ - гомоморфизм группы $G$ в группу $G^{*}$. Тогда справедливы следующие утверэсдения:

1. Ядро гомоморфизма $\varphi$ является нормальным делителем группы $G$.

2. Если $H$ - ядро гомоморфизма $\varphi$, то $G^{*}=\varphi(G)=G / H$, m.е. индекс $|G: H|$ (әде $|\cdot|$ - число әлементов множества) факторгруппь $G / H$ совпадает с порядком $|\varphi(G)|$ грynnвь $\varphi(G)$.

В силу предложения 2 для построения нормального делителя группы $G_{k}$ необходимо построить гомоморфизм группы $G_{k}$ в некоторую группу $G^{*}$. Если при этом $G^{*}-$ конечная группа, то ядро построенного гомоморфизма является нормальным делителем конечного индекса (см. [5]).

В этом разделе мы построим гомоморфизмы группы $G_{k}$ на счетные группы.

Пусть $N_{k}=\{1,2, \ldots, k+1\}$. Зафиксируем $M \subseteq N_{k}$ такое, что $|M|>1$. Пусть отображение $\pi_{M}:\left\{a_{1}, a_{2}, \ldots, a_{k+1}\right\} \rightarrow\left\{a_{i}, i \in M\right\} \cup\{e\}$ определено следуюшим образом:

$$
\pi_{M}\left(a_{i}\right)= \begin{cases}a_{i}, & \text { если } i \in M, \\ e, & \text { если } i \notin M,\end{cases}
$$


где $e$ - единичный элемент группы $G_{k}$.

Обозначим через $G_{M}$ свободное произведение циклических групп $\left\{e, a_{i}\right\}, i \in M$. Ясно, что $G_{M}$ является групповым представлением дерева Кэли $\Gamma^{|M|-1}$.

Для $M \subseteq N_{k}$ отображение $f_{M}: G_{k} \rightarrow G_{M}$ определим следуюшим образом:

$$
f_{M}(x)=f_{M}\left(a_{i_{1}} a_{i_{2}} \ldots a_{i_{n}}\right)=\pi_{M}\left(a_{i_{1}}\right) \pi_{M}\left(a_{i_{2}}\right) \ldots \pi_{M}\left(a_{i_{n}}\right) .
$$

Положим $H_{M}=\left\{x \in G_{k}: f_{M}(x)=e\right\}$.

ПРЕДЛОЖЕНИЕ 3 . Для произвольных $M \subseteq N_{k},|M|>1$, справедливы следующие утверждения:

a) $f_{м}$ является гомоморфизмом;

б) если $x, y \in G_{k}$ - соседние вершинь, то $f_{M}(x)$ и $f_{M}(y)$ либо являются соседними, либо $f_{M}(x)=f_{M}(y)$;

в) $H_{M}$ является нормальным делителем бесконечного индекса группы $G_{k}$.

ДокАЗАТЕЛЬСтво. Если $a_{i} a_{j}=e$ (т.е. $i=j$ ), то $\pi_{M}\left(a_{i}\right) \pi_{M}\left(a_{j}\right)=e$. Отсюда, очевидно, следует утверждение "а"

Пусть $x, y$ - соседние вершины, т.е. $y=x a_{m}$ для некоторого $m \in N_{k}$, тогда по определению

$$
f_{M}(y)=\left\{\begin{array}{lll}
f_{M}(x) a_{m}, & \text { если } m \in M, \\
f_{M}(x), & \text { если } m \notin M .
\end{array}\right.
$$

Отсюда следует утверждение "б".

Утверждение "в" следует из утверждения "а" в силу предложения 2. Предложение доказано.

Следуюшее утверждение дает возможность описать новые нормальные делители бесконечного индекса.

ПРЕДЛОЖЕнИЕ 4. 1. $H_{A} \neq H_{B}$ для любых $A \neq B \subseteq N_{k}$.

2. Для любих $A_{1}, A_{2}, \ldots, A_{n} \in N_{k}$ имеет место равенство

$$
\bigcap_{i=1}^{n} H_{A_{i}}=H_{\bigcap_{i=1}^{n} A_{i}}
$$

Более того, $H_{\bigcap_{i=1}^{n} A_{i}}$ является нормальныцм делителем индекса 1, если $\left|\bigcap_{i=1}^{n} A_{i}\right|=0$, индекса 2 , если $\left|\bigcap_{i=1}^{n} A_{i}\right|=1$, и бесконечного индекса, если $\left|\bigcap_{i=1}^{n} A_{i}\right|>1$.

Доказательство. 1. Пусть $A \neq B \subseteq N_{k}$. Докажем, что $H_{A} \neq H_{B}$. Существует такое $i_{0} \in N_{k}$, которое принадлежит только одному из множеств $A$ и $B$, в противном случае $A=B$. Для определенности пусть $i_{0} \in A$ и $i_{0} \notin B$. Тогда элемент $a_{i_{0}} \notin H_{A}$, поскольку $f_{A}\left(a_{i_{0}}\right)=a_{i_{0}} \neq e$ и $a_{i_{0}} \in H_{B}$, т.е. $f_{B}\left(a_{i_{0}}\right)=e$. Следовательно, $H_{A} \neq H_{B}$.

2. Утверждение пункта 2 очевидно по построению $H_{A}$. Предложение доказано.

Пусть $H$ - некоторая подгруппа бесконечного индекса и $G_{k} / H=\left\{H=H_{0}, H_{1}\right.$, $\left.H_{2}, \ldots\right\}$ - факторгруппа. Обозначим

$$
\begin{gathered}
S(x)=\left\{y \in G_{k}: y=x a_{i}, i=1,2, \ldots, k+1\right\}, \\
q_{i}(x)=\left|S(x) \cap H_{i}\right|, \quad Q(x)=\left\{q_{i}(x)\right\}_{i=1}^{\infty} .
\end{gathered}
$$




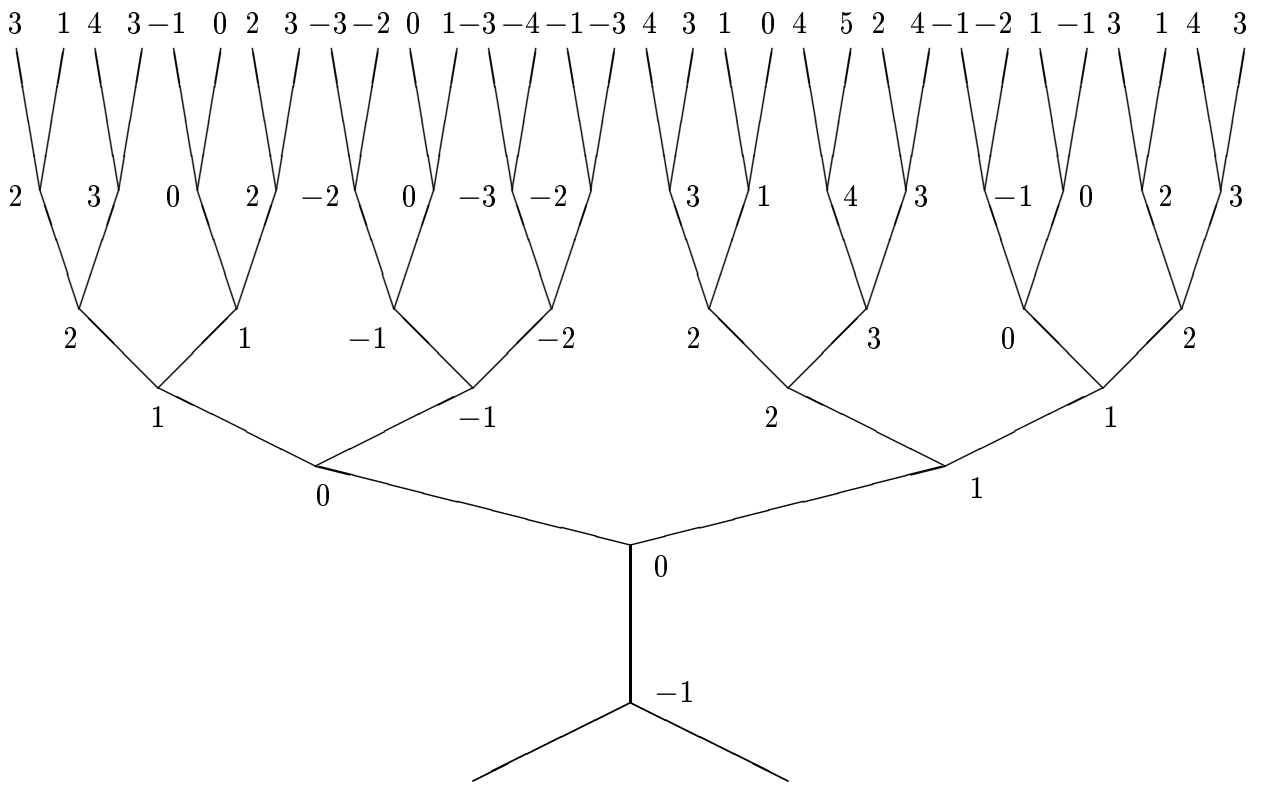

ПРЕДЛОЖЕНИЕ 5. Для любого $x \in G_{k}$ существует отображсение $\tau_{x}: N \rightarrow N$ maкое, что

$$
\tau_{x} Q(e)=Q(x)
$$

әде $\tau_{x} Q(e)=\left\{q_{\tau(i)}(e)\right\}_{i=1}^{\infty}$.

ДокАЗАТЕЛЬСтво. Заметим, что $\left|\left\{i: q_{i}(x) \neq 0\right\}\right|<k+2$ для любого $x \in G_{k}$. Далее доказательство аналогично доказательству теоремы 3 работы [6].

ПримеР. Пусть $M=\{i, j\}, i \neq j \in N_{k}$. Тогда

$$
H_{M}=H_{i j}=\left\{x \in G_{k}: f_{M}(x)=e\right\} .
$$

Рассмотрим факторгруппу

$$
G_{k} / H_{M}=\left\{H_{M}, H_{M}\left(a_{i}\right), H_{M}\left(a_{j}\right), H_{M}\left(a_{i} a_{j}\right), H_{M}\left(a_{j} a_{i}\right), \ldots\right\},
$$

где $H_{M}(y)=\left\{x \in G_{k}: f_{M}(x)=y\right\}, y \in G_{M}$. Введем обозначения

$$
\begin{aligned}
H_{n} & =H_{M}(\underbrace{a_{i} a_{j} \ldots}_{n}), \\
H_{0} & =H_{M}, \\
H_{-n} & =H_{M}(\underbrace{a_{j} a_{i} \ldots}_{n}) .
\end{aligned}
$$

В этих обозначениях факторгруппу $G_{k} / H_{i j}$ можно переписать в виде

$$
G_{k} / H_{i j}=\left\{\ldots, H_{-2}, H_{-1}, H_{0}, H_{1}, H_{2}, \ldots\right\}
$$


Разбиение дерева Кэли $\Gamma^{2}$ относительно $H_{13}$ показано на рисунке (элементы класса $H_{i}$, $i \in \mathbb{Z}$, обозначены просто через $i$ ). В этом случае

$$
\begin{aligned}
Q(e)= & \left\{\ldots, q_{-3}(e)=0, q_{-2}(e)=0, q_{-1}(e)=1, q_{0}(e)=1,\right. \\
& \left.q_{1}(e)=1, q_{2}(e)=0, q_{3}(e)=0, \ldots\right\} .
\end{aligned}
$$

Заметим, что если $x \in H_{i}, i \in \mathbb{Z}$, то

$$
\begin{aligned}
Q(x)= & \left\{\ldots, q_{i-3}(x)=0, q_{i-2}(x)=0, q_{i-1}(x)=1,\right. \\
& \left.q_{i}(x)=1, q_{i+1}(x)=1, q_{i+2}(x)=0, q_{i+3}(x)=0, \ldots\right\} .
\end{aligned}
$$

В качестве $\tau_{x}, x \in H_{i}$, можно взять $\tau_{x}(j)=j+i, j \in \mathbb{Z}$.

\section{3. СЧЕТНО-ПЕРИОДИЧЕСКИЕ МЕРЫ}

Неоднородная модель Изинга на дереве Кэли $G_{k}$ задается гамильтонианом

$$
H(\sigma)=-\sum_{\langle x, y\rangle: x, y \in G_{k}} J_{x y} \sigma(x) \sigma(y),
$$

где суммирование ведется по всем парам ближайших соседей $\langle x, y\rangle$ и $J_{x y} \in \mathbb{R}, \sigma(x) \in$ $\{-1 ; 1\}$ для любых $x, y \in G_{k}$.

Стандартным образом каждой предельной гиббсовской мере $\mu$ на $\Omega=\{-1 ; 1\}^{G_{k}}$ сопоставляется совокупность величин

$$
\mathbf{h}=\left\{h_{x}, x \in G_{k}\right\} \in \Lambda=\left\{\mathbf{h}: h_{x}=\sum_{y \in S_{1}(x)} f\left(h_{y} ; \theta_{x y}\right)\right\}
$$

где $f(h ; \theta)=\operatorname{arcth}(\theta$ th $h),-1<\theta_{x y}=\operatorname{th}\left(\beta J_{x y}\right)<1, S_{1}(x)$ - множество прямых потомков точки $x \in G_{k}$ (см. [14]).

В работе [7] при $J_{x y}=J$ для любых $x, y \in G_{k}$ доказано, что сушествуют три постоянные функции $\mathbf{h}=\left\{h_{x}=\right.$ const,$\left.x \in G_{k}\right\} \in \Lambda$ и несчетное число непостоянных функций из $\Lambda$. В работе [6] для гамильтониана (1) доказано сушествование трех периодических (принимаюших конечное число значений) функций из $\Lambda$. В работе [8] построено несчетное число элементов множества $\Lambda$ с помощью функций, определенных в [6].

В настоящей работе мы построим периодические функции из $\Lambda$, область значений которых представляет собой счетное множество.

ОПрЕДЕЛЕнИЕ 1. Функция $\mathbf{h}=\left\{h_{x}, x \in G_{k}\right\}$ называется $H_{0}$-периодической (где $H_{0}$ - нормальный делитель группы $G_{k}$ ), если

$$
h_{y x}=h_{x} \quad \forall x \in G_{k}, \quad y \in H_{0} .
$$


ОПреДЕЛЕниЕ 2. Гиббсовскую меру назовем $H_{0}$-периодической, если она соответствует $H_{0}$-периодической совокупности величин $\mathbf{h}=\left\{h_{x}, x \in G_{k}\right\}$. Если при этом $H_{0}=$ $G_{k}$, то мера называется трансляционно-инвариантной. Если $H_{0}$-нормальный делитель бесконечного индекса, то меру назовем счетно-периодической.

Таким образом, для описания счетно-периодических гиббсовских мер необходимо построить $H_{0}$-периодические элементы множества $\Lambda$ для нормального делителя $H_{0}$ бесконечного индекса.

Не ограничивая обшности, положим $k=2$. Рассмотрим задачу описания $H_{i j}$-периодических функции (см. пример в разделе 2). В этом случае удается явно выписать вид неизвестной функции $\mathbf{h}$.

Предположим, что

$$
\begin{aligned}
& h_{x}=h_{i}, \quad \text { если } \quad x \in H_{i} . \\
& \theta_{x y}=\theta_{i, j}, \quad \text { если } \quad x \in H_{i}, \quad y \in H_{j} .
\end{aligned}
$$

Тогда в силу предложений 3,4 функциональное уравнение

$$
h_{x}=\sum_{y \in S_{1}(x)} f\left(h_{y} ; \theta_{x y}\right)
$$

имеет вид (см. рисунок):

$$
\begin{aligned}
& h_{n}=f\left(h_{n} ; \theta_{n, n}\right)+f\left(h_{n+1} ; \theta_{n, n+1}\right), \\
& h_{n}=f\left(h_{n} ; \theta_{n, n}\right)+f\left(h_{n-1} ; \theta_{n, n-1}\right), \\
& h_{n}=f\left(h_{n+1} ; \theta_{n, n+1}\right)+f\left(h_{n-1} ; \theta_{n, n-1}\right),
\end{aligned}
$$

где $n \in \mathbb{Z}$. Легко заметить, что $h_{n} \equiv 0$ есть решение системы (3) независимо от значений параметров $\theta_{i, j}$. Также заметим, что если сушествует $n_{0}$ такое, что $h_{n_{0}}=0$, то отсюда следует, что $h_{n} \equiv 0$.

Теперь выберем параметры $\theta_{i, j}$ так, чтобы система (3) имела ненулевые решения. При $n=0$ из (3) имеем

$$
\begin{aligned}
& h_{0}=f\left(h_{0} ; \theta_{0,0}\right)+f\left(h_{1} ; \theta_{0,1}\right), \\
& h_{0}=f\left(h_{0} ; \theta_{0,0}\right)+f\left(h_{-1} ; \theta_{0,-1}\right), \\
& h_{0}=f\left(h_{1} ; \theta_{0,1}\right)+f\left(h_{-1} ; \theta_{0,-1}\right) .
\end{aligned}
$$

Из этой системы следует

$$
f\left(h_{-1} ; \theta_{0,-1}\right)=f\left(h_{0} ; \theta_{0,0}\right)=f\left(h_{1} ; \theta_{0,1}\right) .
$$

Следовательно,

$$
\theta_{0,-1} \text { th } h_{-1}=\theta_{0,0} \text { th } h_{0}=\theta_{0,1} \text { th } h_{1},
$$

откуда при $\theta_{0,-1} \neq 0, \theta_{0,1} \neq 0$ получим

$$
\begin{aligned}
h_{0} & =2 f\left(h_{0} ; \theta_{0,0}\right), \\
h_{1} & =f\left(h_{0} ; \frac{\theta_{0,0}}{\theta_{0,1}}\right), \\
h_{-1} & =f\left(h_{0} ; \frac{\theta_{0,0}}{\theta_{0,-1}}\right) .
\end{aligned}
$$


Таким образом, $h_{0}$ определяется из уравнения

$$
h_{0}=2 f\left(h_{0} ; \theta_{0,0}\right) \text {. }
$$

Решая уравнение (6) относительно $h_{0}$, получим

$$
h_{0}= \begin{cases}0, & \text { если } \theta_{0,0}<1 / 2, \\ 0, \pm h_{0}^{*}, & \text { если } \theta_{0,0} \geqslant 1 / 2\end{cases}
$$

где $h_{0}^{*}=\ln \left[\left(\theta_{0,0}+\sqrt{2 \theta_{0,0}-1}\right) /\left(1-\theta_{0,0}\right)\right]$. Подставляя эти величины в $(5)$, получим

$$
\begin{aligned}
h_{0}^{*} & = \pm \ln \frac{\theta_{0,0}+\sqrt{2 \theta_{0,0}-1}}{1-\theta_{0,0}} \\
h_{1}^{*} & =f\left( \pm h_{0}^{*} ; \frac{\theta_{0,0}}{\theta_{0,1}}\right), \\
h_{-1} & =f\left( \pm h_{0}^{*} ; \frac{\theta_{0,0}}{\theta_{0,-1}}\right), \\
\theta_{0,0}>\frac{1}{2}, \quad 0 & <\left|\theta_{0,1}\right|<1, \quad 0<\left|\theta_{0,-1}\right|<1 .
\end{aligned}
$$

Таким образом, найдены возможные значения функции $h_{n}$ при $n=-1,0,1$.

Теперь с помошью $h_{0}^{*}, h_{1}^{*}$ определим $h_{2}$. При $n=1$ из (3) получим

$$
\begin{aligned}
& h_{1}^{*}=f\left(h_{1}^{*} ; \theta_{1,1}\right)+f\left(h_{2} ; \theta_{1,2}\right), \\
& h_{1}^{*}=f\left(h_{0}^{*} ; \theta_{1,0}\right)+f\left(h_{1}^{*} ; \theta_{1,1}\right), \\
& h_{1}^{*}=f\left(h_{0}^{*} ; \theta_{1,0}\right)+f\left(h_{2} ; \theta_{1,2}\right) .
\end{aligned}
$$

Следовательно,

$$
f\left(h_{1}^{*} ; \theta_{1,1}\right)=f\left(h_{0}^{*} ; \theta_{1,0}\right)=f\left(h_{2} ; \theta_{1,2}\right)
$$

После несложных вычислений получим

$$
\begin{gathered}
h_{2}=h_{2}^{*}=f\left( \pm h_{0}^{*} ; \frac{\theta_{1,0}}{\theta_{1,2}}\right), \\
\theta_{1,0}=\frac{\operatorname{th}\left(h_{1}^{*} / 2\right)}{\operatorname{th} h_{0}^{*}}, \\
\theta_{1,1}=\frac{\operatorname{th}\left(h_{1}^{*} / 2\right)}{\operatorname{th} h_{1}^{*}}, \\
h_{0}^{*} \neq 0, \quad 0<\left|\theta_{1,2}\right|<1,
\end{gathered}
$$

и $h_{2}^{*}=0$ при $h_{0}^{*}=0$.

Таким образом, определены три возможных значения $h_{n}$ при $n=2$. 
Теперь с помощью $h_{1}^{*}, h_{2}^{*}$ можно определить $h_{3}$ и т.д. Используя метод индукции по $n \geqslant 1$, можно показать, что

$$
\begin{aligned}
& h_{n+1}=f\left( \pm h_{n}^{*} ; \frac{\theta_{n, n}}{\theta_{n, n+1}}\right), \\
& \theta_{n, n-1}=\frac{\operatorname{th}\left(h_{n}^{*} / 2\right)}{\operatorname{th} h_{n-1}^{*}}, \\
& \theta_{n, n}=\frac{\operatorname{th}\left(h_{n}^{*} / 2\right)}{\operatorname{th} h_{n}^{*}}, \\
& h_{n-1}^{*} \neq 0, \quad 0<\left|\theta_{n, n+1}\right|<1 .
\end{aligned}
$$

Аналогично при $n \leqslant-1$ имеем

$$
\begin{aligned}
& h_{n-1}=f\left( \pm h_{n}^{*} ; \frac{\theta_{n, n}}{\theta_{n, n-1}}\right), \\
& \theta_{n, n+1}=\frac{\operatorname{th}\left(h_{n}^{*} / 2\right)}{\operatorname{th} h_{n+1}^{*}}, \\
& \theta_{n, n}=\frac{\operatorname{th}\left(h_{n}^{*} / 2\right)}{\operatorname{th} h_{n}^{*}}, \\
& h_{n+1}^{*} \neq 0, \quad 0<\left|\theta_{n, n-1}\right|<1 .
\end{aligned}
$$

Заметим, что на параметры $\theta_{n, n+1} \neq 0, n \geqslant 0$, и $\theta_{n, n-1} \neq 0, n \leqslant 0$, никаких условий не налагается. Поэтому можно выбрать их так, чтобы $h_{n} \neq h_{m}$ для любых $n \neq m$. Таким образом, построены три функции $h_{n}=0, \pm h_{n}^{*}, n \in \mathbb{Z}$, которые удовлетворяют функциональному уравнению (2). Обозначим через $\mu_{0}, \mu_{ \pm}$соответствуюшие им предельные гиббсовские меры.

Таким образом, доказана

Теорема 1. Для неоднородной модели Изинга существует непустое подмножество множсества параметров $J_{x y}, x, y \in V$, такое, что для каждой точки этого подмнохества существуют три счетно-периодические меры.

Заметим, что мера $\mu_{0}$ трансляционно-инвариантна.

\section{4. ОПИСАНИЕ НОВЫХ ПРЕДЕЛЬНЫХ ГИББСОВСКИХ МЕР ДЛЯ МОДЕЛИ ИЗИНГА}

В данном разделе, используя меры $\mu_{0}, \mu_{ \pm}$, докажем, что система уравнений (3) имеет несчетное число непериодических решений при тех же условиях на параметры модели, при которых сушествуют меры $\mu_{0}, \mu_{ \pm}$.

Рассмотрим произвольный бесконечный путь $\pi=\left\{x^{0}=x_{0}, x_{1}, x_{2}, \ldots\right\}$ на дереве Кэли. Каждому бесконечному пути на дереве Кэли второго порядка соответствует вешественное число $t \in[0 ; 3 / 2]$ (см. [8]). Пусть $\pi_{1}=\left\{x^{0}=x_{0}, x_{1}, x_{2}, \ldots\right\}$ и $\pi_{2}=\left\{x^{0}=\right.$ $\left.y_{0}, y_{1}, y_{2}, \ldots\right\}$ - бесконечные пути. Сопоставим путям $\pi_{1}, \pi_{2}$ совокупность чисел $\mathbf{h}^{\pi_{1} \pi_{2}}=$ $\left\{h_{x}^{\pi_{1} \pi_{2}}, x \in V\right\}$, удовлетворяющих уравнению $(2)$. 
Путями $\pi_{1}, \pi_{2}$ дерево Кэли $\Gamma^{2}$ разбивается на три компоненты $\Gamma_{-}^{2}, \Gamma_{0}^{2}, \Gamma_{+}^{2}$, когда пути не совпадают, и на две компоненты $\Gamma_{-}^{2}, \Gamma_{+}^{2}$, когда они совпадают (см. [8]). Рассмотрим совокупность чисел $\mathbf{h}^{\pi_{1} \pi_{2}}$, которая определяется следующим образом:

$$
h_{x}^{\pi_{1} \pi_{2}}= \begin{cases}-h_{i}^{*}, & \text { если } x \in \Gamma_{-}^{2} \cap H_{i}, \\ 0, & \text { если } x \in \Gamma_{0}^{2}, \\ h_{i}^{*}, & \text { если } x \in \Gamma_{+}^{2} \cap H_{i},\end{cases}
$$

где $h_{i}^{*}, i \in \mathbb{Z}$, определено формулами (9), (10).

ТеОрема 2. ДЛя любых бесконечных путей $\pi_{1}, \pi_{2}$ существует единственная совокупность чисел $\mathbf{h}^{\pi_{1} \pi_{2}}$, удовлетворяющих соотношениям (2) и (11).

ДокАЗАТЕЛЬСтво аналогично доказательству теоремы 3 работы [8].

Пусть путям $\pi_{1}, \pi_{2}$ соответствует пара $(t, s) \in[0 ; 3 / 2] \times[0 ; 3 / 2]$. Стандартным образом можно доказать (см. [5]-[9]), что совокупности чисел $\mathbf{h}^{\pi_{1}(t) \pi_{2}(s)}$ различны при различных $(t, s) \in D=\{(u, v) \in[0 ; 3 / 2] \times[0 ; 3 / 2]: u \leqslant v\}$. Обозначим через $\mu(t, s)$ гиббсовскую меру, соответствуюшую совокупности величин $\mathbf{h}^{\pi_{1}(t) \pi_{2}(s)},(t, s) \in D$. Таким образом, справедлива следующая

ТЕОРема 3. Для любой точки $(t, s) \in D$ существует единственная гиббсовская мера $\mu(t, s)$, и при әтом

$$
\mu(0,0)=\mu_{+}, \quad \mu\left(0, \frac{3}{2}\right)=\mu_{0}, \quad \mu\left(\frac{3}{2}, \frac{3}{2}\right)=\mu_{-} .
$$

Поскольку меры $\mu(t, s)$ различны при различных $(t, s) \in D$, мы получаем континуум предельных гиббсовских мер. Заметим, что эти меры не совпадают с известными мерами для модели Изинга.

\section{5. ОБСУЖДЕНИЕ}

Широко распространена (см., например, [1]-[4]) формулировка модели Изинга, согласно которой каждому узлу $x$ решетки сопоставляется переменная $\sigma(x)$, принимающая численные значения +1 или -1 .

Если "объекты", связанные с узлами $x$ и $x^{\prime}$, находятся в одном состоянии, то $\sigma(x) \sigma\left(x^{\prime}\right)=+1$, а если в разных, то $\sigma(x) \sigma\left(x^{\prime}\right)=-1$. Ясно, что в такой интерпретации понятие "объекта", связанного с узлом $x$, может трактоваться весьма широко. Это могут быть, например, магнитньй момент иона в кристалле, имеюший два возможных направления [15], или атомы двух сортов в бинарном сплаве [16] (значение $\sigma(x)=+1$ соответствует занятию $x$-го узла атомом одного сорта, а $\sigma(x)=-1$ - атомом другого сорта). Другие интерпретации модели Изинга связаны с исследованием явления адсорбции на поверхности [17], плавлением ДНК [18], теорией решеточного газа [19] и цельм рядом других вопросов теории фазового перехода типа порядок-беспорядок.

Благодаря простоте формулировки, а также точным решениям, полученным для $k \geqslant 1$ на $\Gamma^{k}$, модель Изинга играет важную роль в теории фазовых переходов.

Из условия $\theta_{x y}=\theta_{i, j}, x \in H_{i}, y \in H_{j}$, следует, что

$$
J_{x y}=J_{i, j}, \quad \text { если } \quad x \in H_{i}, \quad y \in H_{j} .
$$


Таким образом, значения $J_{x y}$ зависят только от классов принадлежности $x$ и $y$. Фактически гамильтониан (1) рассматривается для таких $J_{x y}$, которые удовлетворяют условию (12). Формулы (9) и (10) дают воможность явно вычислить вероятности событий $\sigma(x)=a, a=-1,1$ :

$$
P\{\sigma(x)=a\}=\frac{e^{a h_{x}}}{2 \operatorname{ch} h_{x}} .
$$

В нашем предположении $h_{x}=h_{i}$, если $x \in H_{i}, i \in \mathbb{Z}$, т.е. вероятность (13) зависит только от классов принадлежности $x$.

Можно также явно вычислить средние $\langle\sigma(x)\rangle$ по каждой из мер $\mu_{0}, \mu_{ \pm}$:

$$
\langle\sigma(x)\rangle_{\mu_{0}}=\text { th } 0=0, \quad\langle\sigma(x)\rangle_{\mu_{ \pm}}= \pm \operatorname{th} h_{x} .
$$

Например,

$$
\langle\sigma(e)\rangle_{\mu_{0}}=0, \quad\langle\sigma(e)\rangle_{\mu_{ \pm}}= \pm \frac{2 \theta_{0,0}-1+\sqrt{2 \theta_{0,0}-1}}{\theta_{0,0}^{2}+\sqrt{2 \theta_{0,0}-1}} .
$$

Для рассматриваемой модели на $\Gamma^{2}$ дано точное решение, т.е. найдена критическая температура $T_{\mathrm{c}}=J_{0,0} / \operatorname{arcth} 0.5$ (которая есть решение уравнения $\theta_{0,0}=0.5$ ) такая, что

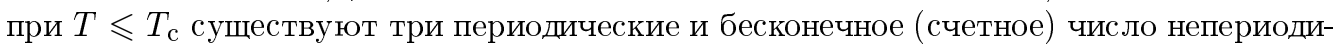
ческих мер. Различные гиббсовские меры соответствуют различным фазам системы. В этом случае существуют фазовые переходы.

В случае $T_{\mathrm{c}}<T$ предельная мера единственна, ей соответствует единственная чистая фаза системы (см., например, [1]), т.е. нет фазовых переходов.

Заметим, что построенные в нашем случае фазы отличаются от известных фаз этой модели тем, что они соответствуют периодическим мерам Гиббса со счетным числом значений.

\section{Список литературы}

[1] Я. Г. Синай. Теория фазовых переходов. Строгие результаты. М.: Наука, 1980.

[2] К. Престон. Гиббсовские состояния на счетных множествах. М.: Мир, 1977.

[3] В. А. Мальишев, Р. А. Минлос. Гиббсовские случайные поля. М.: Наука, 1985.

[4] Д. Рюәль. Статистическая механика. Строгие результаты. М.: Мир, 1971.

[5] Н. Н. Ганиходжаев, У. А. Розиков. ТМФ. 1997. Т. 111. № 1. С. 109-117.

[6] У. А. Розиков. ТМФ. 1997. Т. 112. № 1. С. 170-175.

[7] П. М. Блехер, Н. Н. Ганиходжаев. Теория вероятн. и ее примен. 1990. Т. 35. № 2. C. $220-230$.

[8] У. А. Розиков. ТМФ. 1999. Т. 118. № 1. С. 95-104.

[9] У. А. Розиков. Сиб. матем. журн. 1998. Т. 39. № 2. С. 427-435.

[10] Н. Н. Ганиходжаев, У. А. Розиков. Матем. сб. 1999. Т. 190. № 2. С. 31-42.

[11] Н. Н. Ганиходжсаев. ДАН РУз. 1994. № 5. С. 3-6.

[12] N. N. Ganikhodjaev, U. A. Rozikov. Osaka J. Math. 2000. V. 37. № 2. P. 373-383.

[13] А. Г. Курош. Теория групп. М.: Наука, 1967.

[14.] S. Katsura, M. Takizava. Progr. Theor. Phys. 1974. V. 51. P. 82-98.

[15] L. J. de Jongh, A. R. Miedema. Adv. Phys. 1974. V. 23. P. 397-413.

[16] Р. Фейнман. Статистическая механика. М.: Мир, 1978.

[17] P. W. Kasteleyn. Phase transitions. In: Fundamental Problems in Statistical Mechanics. II. Proc. of 2nd NUFFIC Int. Summer Course (Noordwijk, The Netherlands, 1967). New York, 1968.

[18] B. Zimm, P. Doty, K. Iso. Proc. Nat. Acad. Sci. USA. 1959. V. 45. P. 160.

[19] К. Хуанг. Статистическая механика. М.: Мир, 1966. 